\title{
Diversity, conservation, potential uses, and alien species of lowland plants of Batang Toru, Sumatra, Indonesia
}

\author{
HIRMAS F. PUTRA ${ }^{1}$, WENDY A. MUSTAQIM ${ }^{2,3,4}$ \\ ${ }^{1}$ Department of Biology, Faculty of Mathematics and Natural Sciences, Institut Pertanian Bogor. Jl. Meranti, IPB University Campus Dramaga, Bogor, \\ 16680, West Java, Indonesia \\ ${ }^{2}$ Department of Biology, Faculty of Engineering, Universitas Samudra. Jl. Prof. Dr. Syarief Thayeb, Meurandeh, Langsa 24416, Aceh, Indonesia. \\ Tel.: +62-641-426534, Fax.: +62-641-426534, ‘email: wendyachmmadm@gmail.com; wamustaqim@unsam.ac.id \\ ${ }^{3}$ Botany Division, Generasi Biologi Indonesia Foundation. Jl. Swadaya Barat No. 4, Semampir, Cerme, Gresik 61171, East Java, Indonesia
}

Manuscript received: 24 February 2021. Revision accepted: 28 February 2021.

\begin{abstract}
Putra HF, Mustaqim WA. 2021. Diversity, conservation, potential uses, and alien species of lowland plants of Batang Toru, Sumatra, Indonesia. Biodiversitas 22: 1580-1591. A recent field inventory of lowland Sumatran plant diversity was carried out in two close sites of forest patches in the Batang Toru area. These are Muara Upu Swamp Forest and Simulak Anjing Hill Forest. This study aimed to investigate the species composition of two isolated lowland Sumatran forest patches on the west coast of the northern part of the island. The total number of species recorded was 181 species with 131 species known from Simulak Anjing Hill Forest, 54 from Muara Upu Swamp Forest, and only three species recorded from both sites. There are three species endemic to Sumatra, seven species listed as threatened according to the IUCN Red List, one species protected by the Indonesian government, and two are important records for Sumatra. Compared to the published literature, 88 species have been reported to have one or more uses, with 63 species have been used as the source of medicines, 19 as sources of fibers, 18 as the source of vegetables, and several other uses. Sixteen alien species were recorded including four species categorized as 100 world's worst invasive species. It can be concluded that both areas are a home for important lowland plants of Sumatra as indicated by the presence of threatened, endemic, or poorly documented species. Besides that, there is also a need for concern about the presence of potential invasive alien species.
\end{abstract}

Keywords: alien plants, endemic, exploration, Sumatra, threatened plants

\section{INTRODUCTION}

Sumatra is the westernmost and the third largest island in Indonesia. This area is also a home for one of the most diverse places on Earth. The tropical rainforest of Sumatra has been included in the UNESCO World Heritage, namely the Gunung Leuser National Park, Kerinci Seblat National Park, and Bukit Barisan Selatan National Park (UNESCO 2020). The most prominent feature of this island is Bukit Barisan Mountains, formed by the collision between the Indian Ocean and Sundaland plate tectonics. This mountain complex stretches from the north to the south ends of the island with various types of vegetation including the Indonesian highest volcanic summit of Mount Kerinci at $3850 \mathrm{~m}$ above sea level. The lowland parts of Sumatra also consist of many vegetation types including the highlythreatened peat swamp forests that are mainly found in the coastal areas of the eastern side (Global Wetlands 2020).

The biodiversity of Sumatra is exceptional both for animals and plants. For plants, this island possibly inhabited by as many as 8391 species with around 1891 or 22.5 percent of them are endemic (Middleton et al. 2019). Many species are very flamboyant such as the giant parasite Rafflesia (Susatya 2011), the tallest flower titan arum Amorphophallus titanum (Yuzammi et al. 2017), and also home of many commercially important dipterocarp species such as Dipterocarpus, Hopea, and Shorea (Ashton
1982). Many other plant groups have high diversity in Sumatra, such as the beautiful forest floor dwellers Impatiens (Balsaminaceae) (Grey-Wilson 1989) and the pitcher plant genus Nepenthes (Nepenthaceae) (Cheek and Jebb 2001).

The lowland ecosystem of Sumatra is among the most threatened ecosystems in the world. Loucks and Whitten (2020) categorized the Sumatran Lowland Rain Forests as a critical or endangered ecoregion, and several important factors contribute to the damage of the ecosystems, such as fires, logging, and forest fires, that have become serious threats to the biodiversity in this area. Any remaining forest ecosystem in the lowland area of Sumatra becomes an important factor in its conservation as they may harbor species threatened with extinction. These often present in the form of fragmented and isolated landscapes within large plantations.

As a starting point to the future conservation efforts of lowland biodiversity of Sumatra, biodiversity inventories are needed to be conducted in poorly explored areas. According to Laumonier et al. (2010), Muara Upu Swamp Forest and Simulak Anjing Hill Forest are located in the vulnerable or endangered eco-floristic sectors. The Simulak Anjing Hill is also home to endemic Thottea tapanuliensis (Mustaqim and Putra 2020a). In the study, we investigate the plant diversity in both areas that represent the isolated fragments of the lowland forest of Sumatra. 


\section{MATERIALS AND METHODS}

\section{Study area}

The study was conducted in Muara Upu swamp forest (MUSF; 142.88 ha) and Simulak Anjing Hills forest (SAHF; 73.12 ha) in Batang Toru, Tapanuli Selatan Regency, North Sumatra Province (Figure 1). Both forests are located in the concession area of palm oil plantations named PT. Perkebunan Nusantara III (or PTPN III) and have been declared as High Conservation Value (HCV) areas. Each MUSF and SAHF represent a different type of vegetation (Figure 2). MUSF consists of degraded lowland peat swamp forest and riverine forest on alluvial at an elevation from 21 to $22 \mathrm{~m}$ above sea level. A part of them has been converted into road and agricultural fields. Some commonly observed plants include Gluta wallichii, Nauclea officinalis, shrubby Pandanus helicopus, and Licuala paludosa. Meanwhile, SAHF consists of lowland isolated hill forest, typically belonging to the lowland mixed dipterocarp forest at an elevation from 70 to $250 \mathrm{~m}$ above sea level. There are two major types of vegetation in SHAF, the less disturbed forest and the secondary vegetation. The less-disturbed forest areas have dense forest with large trees such as Dryobalanops sumatrensis, while in the secondary vegetations, shrub and small trees are the most important, like Macaranga heynei or Endospermum diadenum, which are quite common, in this area.

\section{Data collection}

The botanical survey was conducted using an exploratory method (Rugayah et al. 2004) in Muara Upu and Simulak Anjing Hills in January 2020 to all vascular plants. The identification was carried out in the field by the authors, and several species were also vouchered when the field identification was doubtful. The specimens were then identified in the Laboratory of Ecology and Plant Resources, Department of Biology, IPB University and later deposited to the Herbarium Bogoriense (BO), Research Center for Biology, Indonesian Institute of Sciences, and Herbarium Bandungense (FIPIA), Bandung Institute of Technology (FIPIA) (abbreviations follow Thiers 2020-continuously updated). The species name followed Plants of the World Online databases (POWO 2020). Conservation statuses of the species were acquired from the IUCN Red List (IUCN 2020).

\section{RESULTS AND DISCUSSION}

The total number of plant species recorded was 182 , with 131 species recorded from SAHF and 54 from MUSF (Table 1). There is a high difference in the floristic composition, only three species were recorded from both areas, meaning that 51 species were restricted to MUSF and 128 in SAHF. The largest plant family in SAHF was Dipterocarpaceae (12 spp.), followed by Euphorbiaceae (10 spp.), Fabaceae, and Rubiaceae (each with 7 spp.), while in MUSF, the three largest plant families were Moraceae, Poaceae, and Rubiaceae (each with 4 spp.), followed by Arecaceae (3 spp.) (Figure 3). The striking difference may be in the presence of 12 dipterocarp species in SAHF, while there is none from MUSF (Table 1). Macaranga is the most diverse genus with six species and is the common genus in the secondary vegetations in SAHF.

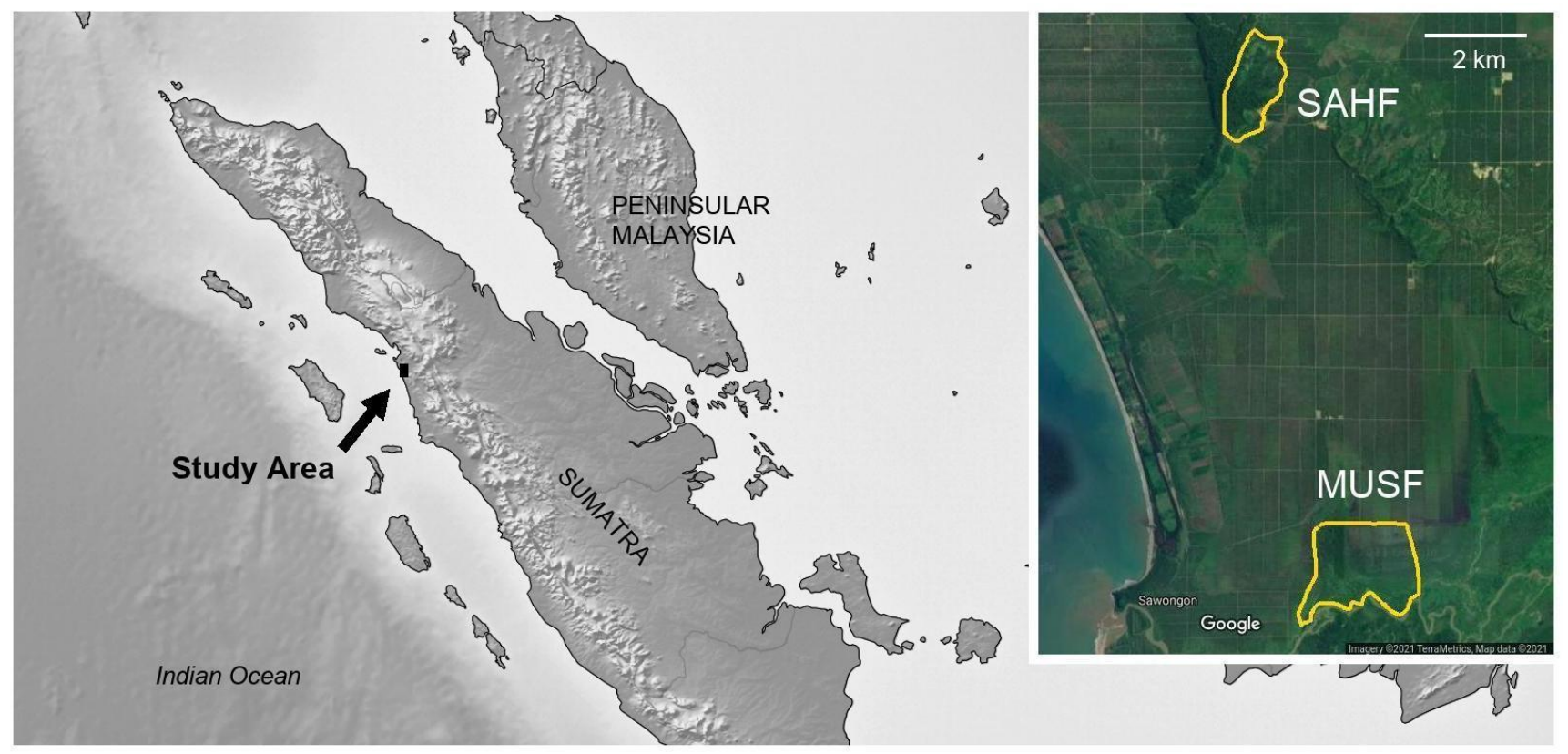

Figure 1. Map of study area in Muara Upu Swamp Forest and Simulak Anjing Hill Forest, Batang Toru, Tapanuli Selatan Regency, North Sumatra Province 

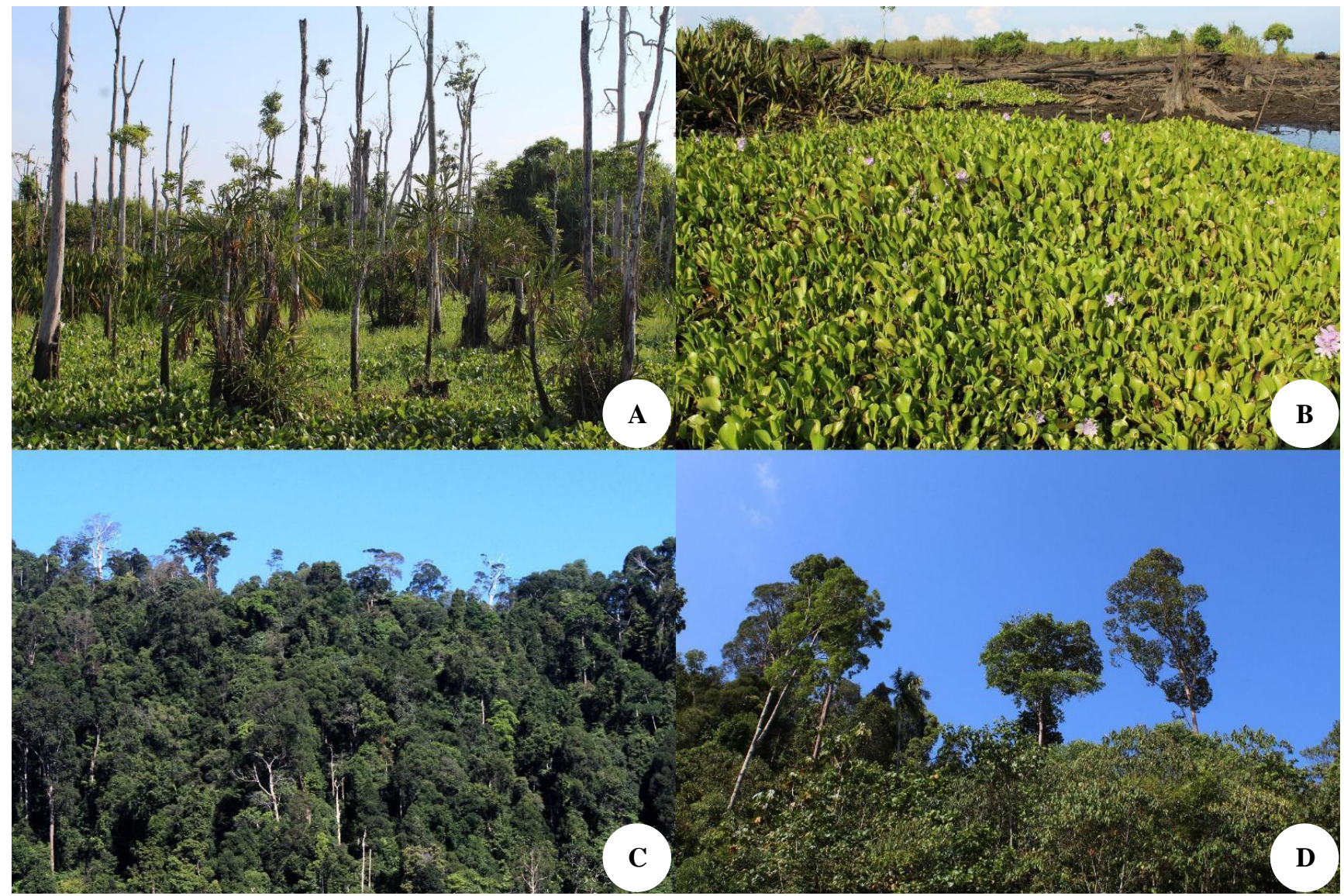

Figure 2. Vegetations in Muara Upu Swamp Forest (A-B) and Simulak Anjing Hill Forest (C-D), Batang Toru, Tapanuli Selatan Regency, North Sumatra Province

\section{Plant life-form}

In terms of plant life form, MUSF is largely composed of herbaceous plant species, while the SAHF is largely composed of trees. This situation has been expected since the plant species in less disturbed part of forest, like in SAHF, will have a higher percentage of trees compared to the degraded forest, like MUSF (Figure 4). The low percentage of herbaceous plants in SAHF is a characteristic of the lowland rain forest of Sumatra (Loucks and Whitten 2020) and could be an indication that past disturbance level in the forested landscape of SAHF was considered low.

\section{Conservation status and protected species}

The conservation status of more than a half number of the total species has not been evaluated by the IUCN (2020) with 97 species or $58.43 \%$. This study will be an important component in the future assessment of all those species. MUSF and SAHF are very specific sites and any data could contribute to the distribution records. The records can be used to calculate the Extent of Occurrence (EOO) and Area of Occupancy (AOO), which are important components in conservation status evaluation (IUCN 2012; IUCNSPC 2019). The other 69 species which have been evaluated consist of 59 species or $85.51 \%$ Least Concern (LC), three Near Threatened (NT), and seven species belong to threatened categories. There are four species listed as Vulnerable (VU) named Aquilaria hirta (Thymelaeaceae) (Figure 5A), Cantleya corniculata (Stemonuraceae) (Figure 5B), and two from Dipterocarpaceae named Dipterocarpus crinitus (Figure 5C), and Dryobalanops sumatrensis (Figure 5D). One dipterocarp species named Vatica perakensis (Figure 5E) is the only species listed as Endangered (EN). The most threatened species are the commercial timber trees Shorea johorensis from the Dipterocarpaceae family (Figure 5F), and one pitcher plant species Nepenthes sumatrana (Nepenthaceae) (Figure 5G), which is also an endemic of Sumatra (Clarke 2001, 2014; Cheek and Jebb 2001). The presence of those threatened plant species indicates the importance of SAHF as a conservation site, and therefore, designation as a High Conservation Value site should be maintained as long as possible. Nepenthes sumatrana or in Indonesian known as 'kantung semar sayap alur' is protected by the Indonesian Government under the Peraturan Pemerintah No. 106/2018. The species was recorded in SAHF on early regenerate vegetation. Only two pitcher plants were observed during the study, and are grown on the steep slope. Another species of pitcher plant, $N$. ampullaria, is also present near the population of $N$. sumatrana. 


\section{Noteworthy plant records}

Acrotrema in North Sumatra confirmed

Acrotrema is a genus belonging to the Dilleniaceae family. This genus consists of around 10 species of mostly Asian mainland inhabitants and only one species extends to Malesia. The only Acrotrema species in Malesia is Acrotrema costatum Jack (Hoogland 1951) (Figs. 5H-I). Hoogland (1951) mentioned the distribution in North Sumatra as questionable as he wrote 'N. Sumatra?'. Therefore, it can be concluded that the occurrence of this species in Sumatra needs to be confirmed. In Simulak Anjing, many individuals of plants belonging to this species have been found (specimen Mustaqim et al. 2335). They belong to Acrotrema due to some characteristics: perennial herbs, amplexicaul petiole, numerous free stamens, and the flat receptacle. Further examinations of the literature have shown that morphological characteristics are matched to the A. costatum (Hoogland 1951). Therefore, it can be concluded that the distribution of $A$. costatum in Sumatra is now confirmed.

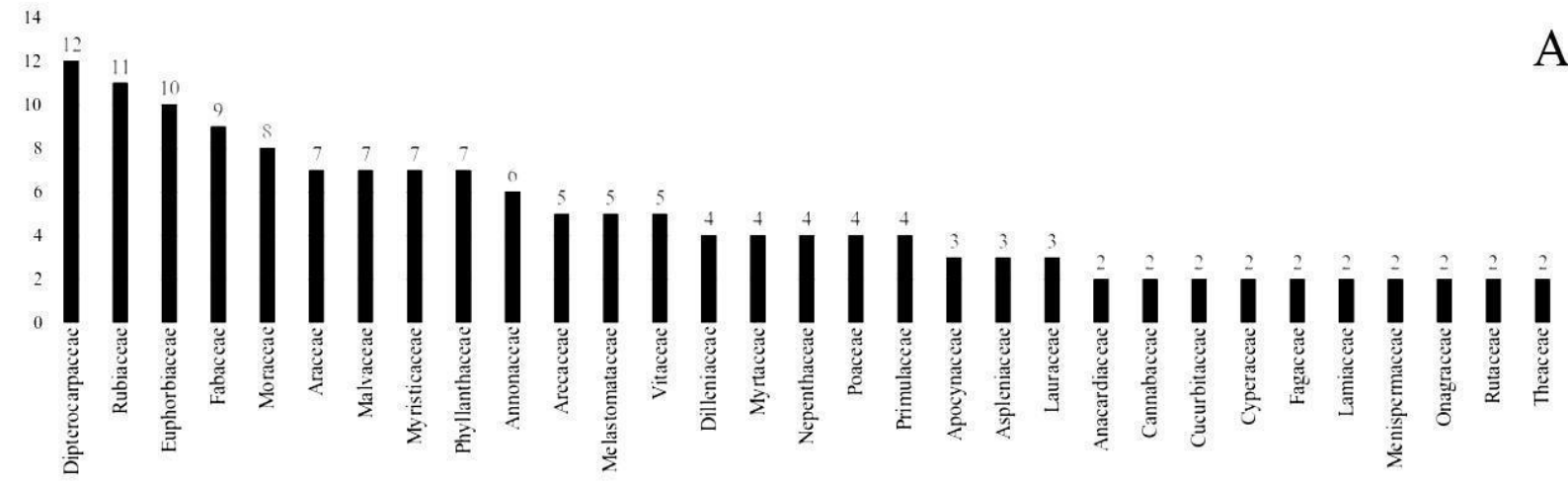

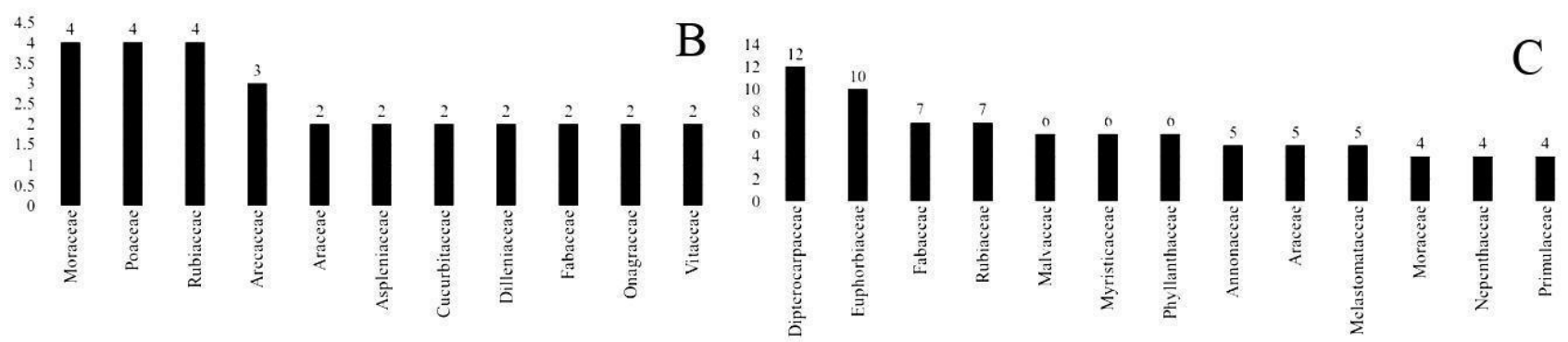

Figure 3. Number of species in largest plant families in both areas (A), Muara Upu Swamp Forest (B), and Simulak Anjing Hill Forest (C), , Batang Toru, North Sumatra, Indonesia
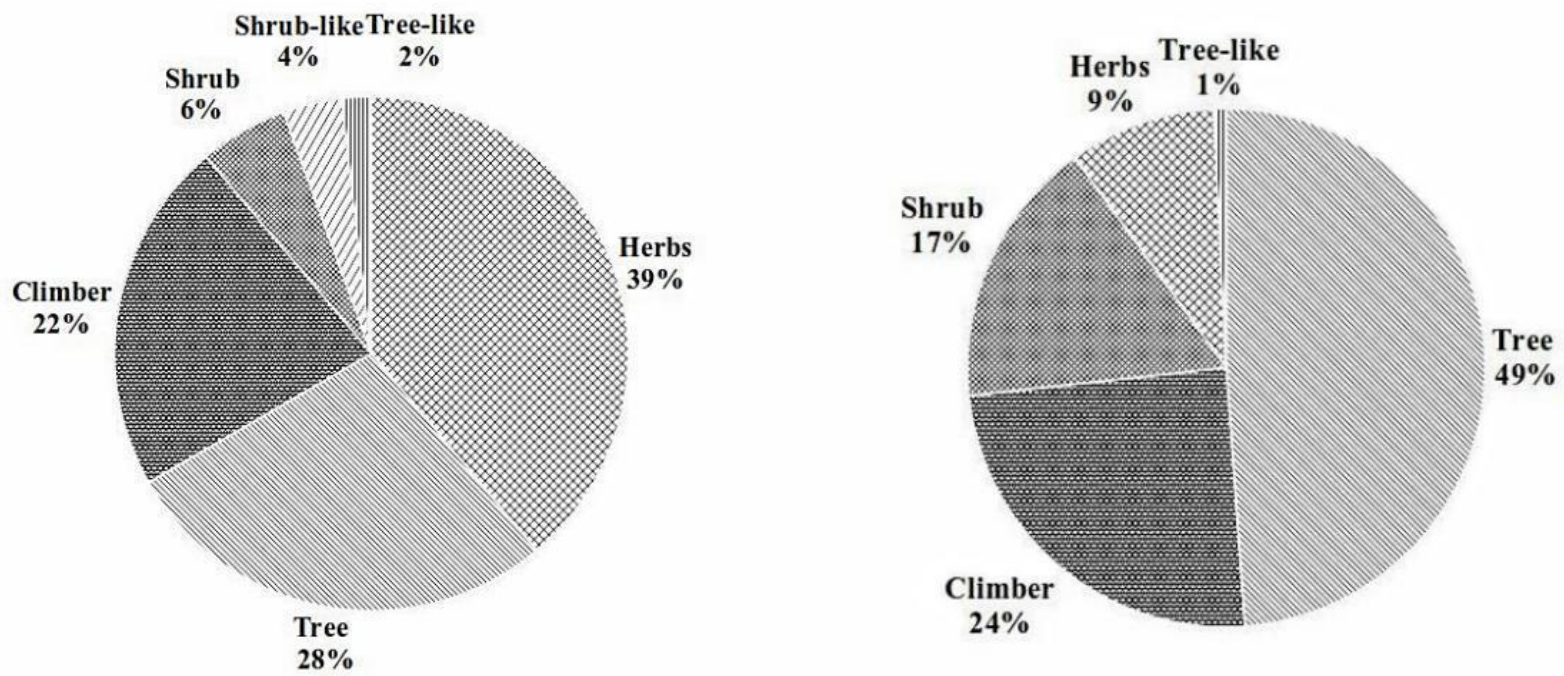

Figure 4. Percentage of life-form of plant species in Muara Upu Swamp Forest and Simulak Anjing Hill Forest, Batang Toru, North Sumatra, Indonesia 
Table 1. List of plant species from Muara Upu and Simulak Anjing Hills, Batang Toru, North Sumatra, Indonesia

\begin{tabular}{|c|c|c|c|c|c|}
\hline Family/species & MU & SA & $\mathbf{L F}$ & IUCN & IDN \\
\hline \multicolumn{6}{|l|}{ Acrostichaceae } \\
\hline Acrostichum aureum $\mathrm{L}$. & $\sqrt{ }$ & & $\mathrm{H}$ & LC & \\
\hline \multicolumn{6}{|l|}{ Anacardiaceae } \\
\hline Gluta wallichii (Hook.f.) Ding Hou & & $\sqrt{ }$ & $\mathrm{T}$ & NE & \\
\hline Mangifera griffithiiHook.f. & & $\sqrt{ }$ & $\mathrm{T}$ & $\mathrm{NE}$ & \\
\hline \multicolumn{6}{|l|}{ Anisophylleaceae } \\
\hline Anisophyllea disticha (Jack) Baill. & & $\sqrt{ }$ & $\mathrm{S}$ & $\mathrm{LC}$ & \\
\hline \multicolumn{6}{|l|}{ Annonaceae } \\
\hline Cananga odorata (Lam.) Hook.f. \& Thomson & $\sqrt{ }$ & & $\mathrm{T}$ & $\mathrm{LC}$ & \\
\hline Dasymaschalon dasymaschalum (Blume) I.M.Turner & & $\sqrt{ }$ & $\mathrm{S}$ & $\mathrm{NE}$ & \\
\hline Goniothalamus macrophyllus (Blume) Zoll. & & $\sqrt{ }$ & $\mathrm{S}$ & $\mathrm{NE}$ & \\
\hline Maasia glauca (Hassk.) Mols, Kessler \& Rogstad & & $\sqrt{ }$ & $\mathrm{T}$ & $\mathrm{NE}$ & \\
\hline Popowia pisocarpa (Blume) Endl. ex Walp. & & $\sqrt{ }$ & $\mathrm{S}$ & $\mathrm{NE}$ & \\
\hline Xylopia cf. oxyantha (Hook.f. \& Thomson) Hook.f. \& Thomson & & $\sqrt{ }$ & $\mathrm{T}$ & - & \\
\hline \multicolumn{6}{|l|}{ Apocynaceae } \\
\hline Alstonia spatulata Blume & & $\sqrt{ }$ & $\mathrm{T}$ & $\mathrm{LC}$ & \\
\hline Dyera costulata (Miq.) Hook.f. & & $\sqrt{ }$ & $\mathrm{T}$ & $\mathrm{LC}$ & \\
\hline Vincetoxicum flexuosum (R.Br.) Kuntze & $\sqrt{ }$ & & $\mathrm{C}$ & $\mathrm{NE}$ & \\
\hline \multicolumn{6}{|l|}{ Araceae } \\
\hline Colocasia esculenta (L.) Schott & $\sqrt{ }$ & & $\mathrm{H}$ & $\mathrm{LC}$ & \\
\hline Homalomena humilis (Jack) Hook.f. & & $\sqrt{ }$ & $\mathrm{H}$ & NE & \\
\hline Pothos scandens L. & & $\sqrt{ }$ & $\mathrm{C}$ & $\mathrm{NE}$ & \\
\hline Rhaphidophora korthalsii Schott & & $\sqrt{ }$ & $\mathrm{C}$ & NE & \\
\hline Schismatoglottis calyptrata (Roxb.) Zoll. \& Moritzi & & $\sqrt{ }$ & $\mathrm{H}$ & $\mathrm{NE}$ & \\
\hline Scindapsus pictus Hassk. & & $\sqrt{ }$ & $\mathrm{C}$ & $\mathrm{NE}$ & \\
\hline Syngonium podophyllum Schott & $\sqrt{ }$ & & $\mathrm{C}$ & $\mathrm{NE}$ & \\
\hline \multicolumn{6}{|l|}{ Arecaceae } \\
\hline Calamus diepenhorstii Miq. & & $\sqrt{ }$ & $\mathrm{C}$ & $\mathrm{NE}$ & \\
\hline Calamus melanochaetes (Blume) Miq. & $\sqrt{ }$ & & $\mathrm{C}$ & $\mathrm{NE}$ & \\
\hline Licuala paludosa Griff. & $\sqrt{ }$ & & SL & $\mathrm{NE}$ & \\
\hline Oncosperma horridum (Griff.) Scheff. & & $\sqrt{ }$ & $\mathrm{TL}$ & NE & \\
\hline Oncosperma tigillarium (Jack) Ridl. & $\sqrt{ }$ & & $\mathrm{TL}$ & $\mathrm{NE}$ & \\
\hline \multicolumn{6}{|l|}{ Aristolochiaceae } \\
\hline Thottea tapanuliensis Mustaqim & & $\sqrt{ }$ & $\mathrm{H}$ & $\mathrm{NE}$ & \\
\hline \multicolumn{6}{|l|}{ Aspleniaceae } \\
\hline Asplenium nidus $\mathrm{L}$. & $\sqrt{ }$ & & $\mathrm{H}$ & $\mathrm{NE}$ & \\
\hline Blechnum orientale $\mathrm{L}$. & & $\sqrt{ }$ & $\mathrm{H}$ & $\mathrm{NE}$ & \\
\hline Stenochlaena palustris (Burm.f.) Bedd. & $\sqrt{ }$ & & $\mathrm{H}$ & $\mathrm{NE}$ & \\
\hline \multicolumn{6}{|l|}{ Asteraceae } \\
\hline Mikania micrantha Kunth. & $\sqrt{ }$ & & $\mathrm{H}$ & - & \\
\hline \multicolumn{6}{|l|}{ Begoniaceae } \\
\hline Begonia isoptera Dryand. ex Sm. & & $\sqrt{ }$ & $\mathrm{H}$ & $\mathrm{NE}$ & \\
\hline \multicolumn{6}{|l|}{ Burseraceae } \\
\hline Dacryodes rugosa (Blume) H.J.Lam & & $\sqrt{ }$ & $\mathrm{T}$ & $\mathrm{LC}$ & \\
\hline \multicolumn{6}{|l|}{ Cannabaceae } \\
\hline Gironniera nervosa Planch. & & $\sqrt{ }$ & $\mathrm{T}$ & $\mathrm{NE}$ & \\
\hline Trema cannabinum Lour. & & $\sqrt{ }$ & $\mathrm{T}$ & $\mathrm{LC}$ & \\
\hline \multicolumn{6}{|l|}{ Celastraceae } \\
\hline Lophopetalum javanicum (Zoll.) Turcz. & $\sqrt{ }$ & & $\mathrm{T}$ & $\mathrm{LC}$ & \\
\hline Clusiaceae & & & & & \\
\hline Garcinia nervosa (Miq.) Miq. & & $\sqrt{ }$ & $\mathrm{T}$ & $\mathrm{NE}$ & \\
\hline Commelinaceae & & & & & \\
\hline Commelina diffusa Zoll. ex C.B.Clarke & $\sqrt{ }$ & & $\mathrm{H}$ & $\mathrm{LC}$ & \\
\hline Connaraceae & & & & & \\
\hline Cnestis palala (Lour.) Merr. & & $\sqrt{ }$ & $\mathrm{C}$ & $\mathrm{NE}$ & \\
\hline Costaceae & & & & & \\
\hline Cheilocostus speciosus (J.Koenig) C.D.Specht & & $\sqrt{ }$ & $\mathrm{H}$ & $\mathrm{LC}$ & \\
\hline Cucurbitaceae & & & & & \\
\hline Melothriapendula $\mathrm{L}$. & $\sqrt{ }$ & & $\mathrm{C}$ & - & \\
\hline Trichosanthes costata Blume & $\sqrt{ }$ & & $\mathrm{C}$ & $\mathrm{NE}$ & \\
\hline Cyperaceae & & & & & \\
\hline Cyperus iria $\mathrm{L}$. & $\sqrt{ }$ & & $\mathrm{H}$ & $\mathrm{NE}$ & \\
\hline Scleria sumatrensis Retz. & & $\sqrt{ }$ & $\mathrm{H}$ & $\mathrm{NE}$ & \\
\hline
\end{tabular}




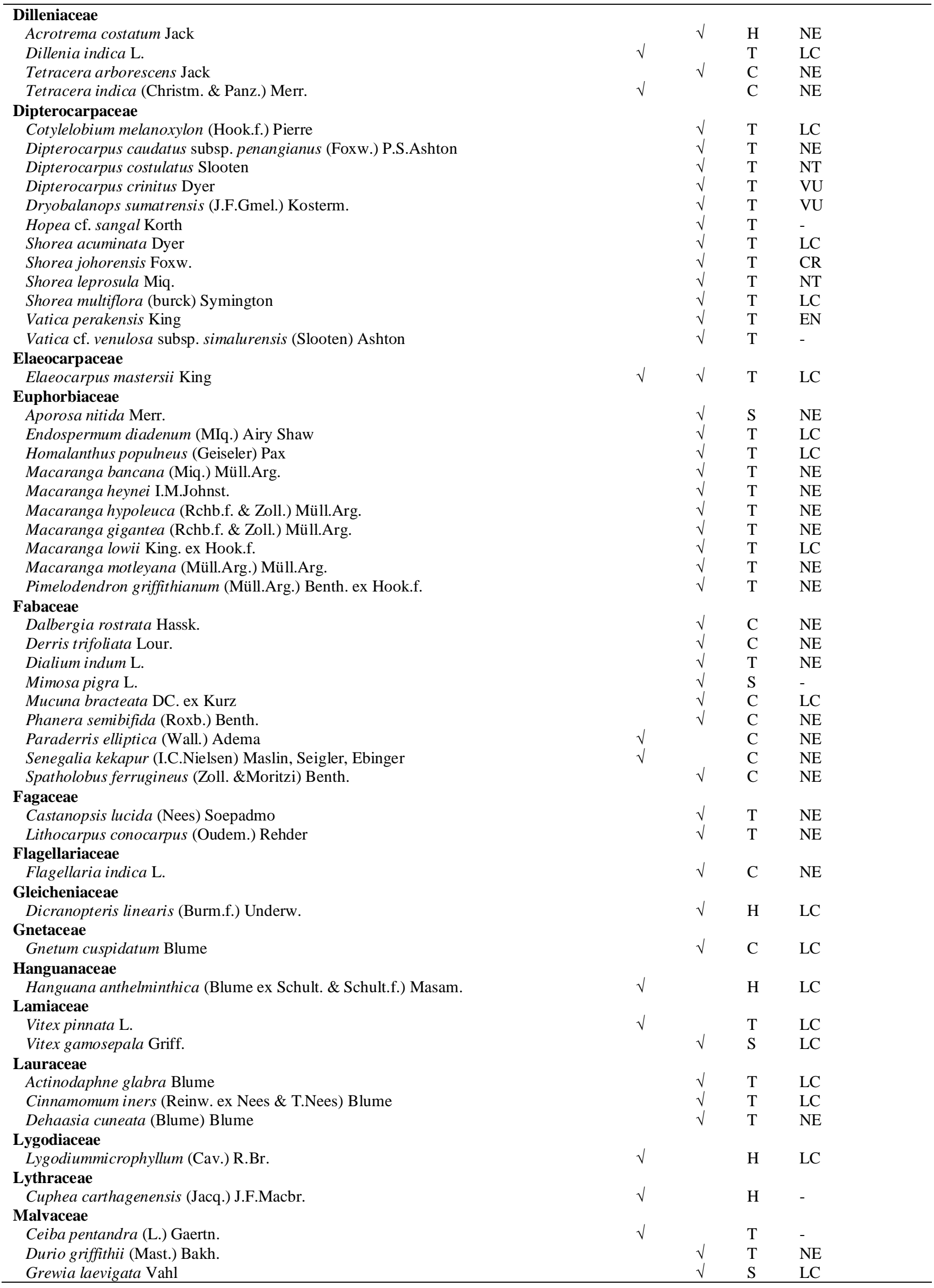




\begin{tabular}{|c|c|c|c|c|c|}
\hline Pterospermum javanicum Jungh. & & $\sqrt{ }$ & $\mathrm{T}$ & LC & \\
\hline Scaphium macropodum (Miq.) Beumée ex K.Heyne & & $\sqrt{ }$ & $\mathrm{T}$ & $\mathrm{LC}$ & \\
\hline Sterculia rubiginosa Vent. & & $\sqrt{ }$ & $\mathrm{T}$ & $\mathrm{NE}$ & \\
\hline Urena lobata L. & & $\sqrt{ }$ & $\mathrm{S}$ & $\mathrm{LC}$ & \\
\hline \multicolumn{6}{|l|}{ Melastomataceae } \\
\hline Clidemia hirta (L.) D.Don & & $\sqrt{ }$ & $\mathrm{S}$ & - & \\
\hline Dissochaeta conica (Bakh.f.) Clausing & & $\sqrt{ }$ & $\mathrm{C}$ & $\mathrm{NE}$ & \\
\hline Macrolenes tuberculata Karton. & & $\sqrt{ }$ & $\mathrm{C}$ & $\mathrm{NE}$ & \\
\hline Melastoma malabathricum $\mathrm{L}$. & $\sqrt{ }$ & $\sqrt{ }$ & $\mathrm{S}$ & $\mathrm{NE}$ & \\
\hline Melastoma penicillatum Naudin & & $\sqrt{ }$ & $\mathrm{S}$ & $\mathrm{NE}$ & \\
\hline \multicolumn{6}{|l|}{ Menispermaceae } \\
\hline Arcangelisia flava (L.) Merr. & & $\sqrt{ }$ & $\mathrm{C}$ & $\mathrm{NE}$ & \\
\hline Cyclea barbata Miers & & $\sqrt{ }$ & $\mathrm{C}$ & $\mathrm{NE}$ & \\
\hline \multicolumn{6}{|l|}{ Moraceae } \\
\hline Artocarpus elasticus Reinw. ex Blume & $\sqrt{ }$ & & $\mathrm{T}$ & $\mathrm{LC}$ & \\
\hline Ficus deltoidea Jack & & $\sqrt{ }$ & $\mathrm{S}$ & $\mathrm{NE}$ & \\
\hline Ficus fistulosa Reinw. ex Blume & $\sqrt{ }$ & & $\mathrm{T}$ & $\mathrm{LC}$ & \\
\hline Ficus grossularioides var. stenoloba Corner & & $\sqrt{ }$ & $\mathrm{T}$ & $\mathrm{LC}$ & \\
\hline Ficus hispida L.f. & $\sqrt{ }$ & & $\mathrm{T}$ & $\mathrm{LC}$ & \\
\hline Ficus microcarpa L.f. & $\sqrt{ }$ & & $\mathrm{T}$ & $\mathrm{LC}$ & \\
\hline Ficus padana Burm.f. & & $\sqrt{ }$ & $\mathrm{T}$ & LC & \\
\hline Ficus punctata Thunb. & & $\sqrt{ }$ & $\mathrm{C}$ & $\mathrm{NE}$ & \\
\hline \multicolumn{6}{|l|}{ Myristicaceae } \\
\hline Horsfieldia grandis (Hook.f.) Warb. & & $\sqrt{ }$ & $\mathrm{T}$ & LC & \\
\hline Horsfieldia irya (Gaertn.) Warb. & $\sqrt{ }$ & & $\mathrm{T}$ & LC & \\
\hline Horsfieldia wallichii (Hook.f. \& Thomson) Warb. & & $\sqrt{ }$ & $\mathrm{T}$ & LC & \\
\hline Knema furfuracea (Hook.f. \& Thomson) Warb. & & $\sqrt{ }$ & $\mathrm{T}$ & $\mathrm{LC}$ & \\
\hline Knema laurina (Blume) Warb. & & $\sqrt{ }$ & $\mathrm{T}$ & $\mathrm{NE}$ & \\
\hline Myristica iners Blume & & $\sqrt{ }$ & $\mathrm{T}$ & $\mathrm{LC}$ & \\
\hline Myristica maxima Warb. & & $\sqrt{ }$ & $\mathrm{T}$ & LC & \\
\hline \multicolumn{6}{|l|}{ Myrtaceae } \\
\hline Rhodamnia cinerea Jack & & $\sqrt{ }$ & $\mathrm{T}$ & LC & \\
\hline Syzygiumpapillosum (Duthie) Merr. \& L.M.Perry & $\sqrt{ }$ & & $\mathrm{T}$ & $\mathrm{NE}$ & \\
\hline Syzygium cf. pycnanthum Merr. \& L.M.Perry & & $\sqrt{ }$ & $\mathrm{T}$ & - & \\
\hline Syzygium zeylanicum (L.) DC. & & $\sqrt{ }$ & $\mathrm{T}$ & $\mathrm{NE}$ & \\
\hline \multicolumn{6}{|l|}{ Nepenthaceae } \\
\hline Nepenthes ampullaria Jack & & $\sqrt{ }$ & $\mathrm{C}$ & LC & \\
\hline Nepenthes gracilis Korth. & $\sqrt{ }$ & $\sqrt{ }$ & $\mathrm{C}$ & LC & \\
\hline Nepenthes mirabilis (Lour.) Druce & & $\sqrt{ }$ & $\mathrm{C}$ & $\mathrm{LC}$ & \\
\hline Nepenthes sumatrana(Miq.) Beck ex Tamin \& M.Hotta & & $\sqrt{ }$ & $\mathrm{C}$ & CR & $\sqrt{ }$ \\
\hline \multicolumn{6}{|l|}{ Nephrolepidaceae } \\
\hline Nephrolepis hirsutula (Forst.) C.Presl & & $\sqrt{ }$ & $\mathrm{H}$ & $\mathrm{NE}$ & \\
\hline \multicolumn{6}{|l|}{ Nymphaeaceae } \\
\hline Nymphaea alba $\mathrm{L}$. & $\sqrt{ }$ & & $\mathrm{H}$ & $\mathrm{LC}$ & \\
\hline \multicolumn{6}{|l|}{ Onagraceae } \\
\hline Ludwigia peruviana (L.) H.Hara & $\sqrt{ }$ & & $\mathrm{H}$ & - & \\
\hline Ludwigia hyssopifolia (G.Don) Exell & $\sqrt{ }$ & & $\mathrm{H}$ & - & \\
\hline \multicolumn{6}{|l|}{ Pandanaceae } \\
\hline Pandanus helicopus Kurz ex Miq. & $\sqrt{ }$ & & SL & $\mathrm{NE}$ & \\
\hline \multicolumn{6}{|l|}{ Pentaphylacaceae } \\
\hline Adinandra dumosa Jack & & $\sqrt{ }$ & $\mathrm{T}$ & LC & \\
\hline \multicolumn{6}{|l|}{ Phyllanthaceae } \\
\hline Antidesma stipulare Blume & & $\sqrt{ }$ & $\mathrm{T}$ & LC & \\
\hline Baccaurea parviflora (Müll.Arg.) Müll.Arg. & & $\sqrt{ }$ & $\mathrm{T}$ & $\mathrm{NE}$ & \\
\hline Baccaurea sumatrana (Miq.) Müll.Arg. & & $\sqrt{ }$ & $\mathrm{T}$ & $\mathrm{NE}$ & \\
\hline Breynia macrantha (Hassk.) Chakrab. \& N.P.Balakr. & & $\sqrt{ }$ & $\mathrm{S}$ & $\mathrm{LC}$ & \\
\hline Breynia racemosa (Blume) Müll.Arg. & $\sqrt{ }$ & & $\mathrm{S}$ & $\mathrm{LC}$ & \\
\hline Glochidion superbum Baill. & & $\sqrt{ }$ & $\mathrm{T}$ & $\mathrm{NE}$ & \\
\hline Phyllanthus lutescens (Blume) Müll.Arg. & & $\sqrt{ }$ & $\mathrm{T}$ & $\mathrm{LC}$ & \\
\hline \multicolumn{6}{|l|}{ Poaceae } \\
\hline Ottochloa nodosa (Kunth) Dandy & $\sqrt{ }$ & & $\mathrm{H}$ & $\mathrm{NE}$ & \\
\hline Paspalum conjugatum P.J.Bergius & $\sqrt{ }$ & & $\mathrm{H}$ & - & \\
\hline Phragmites karka (Retz.) Trin. ex Steud. & $\sqrt{ }$ & & $\mathrm{H}$ & LC & \\
\hline Urochloa mutica (Forssk.) T.Q.Nguyen & $\sqrt{ }$ & & $\mathrm{H}$ & LC & \\
\hline Polygalaceae & & & & & \\
\hline $\begin{array}{l}\text { Polygala paniculata } \mathrm{L} \text {. } \\
\text { Polypodiaceae }\end{array}$ & $\sqrt{ }$ & & $\mathrm{H}$ & - & \\
\hline
\end{tabular}




\begin{tabular}{|c|c|c|c|c|}
\hline Drynaria quercifolia (L.) J.Sm. & $\sqrt{ }$ & & $\mathrm{H}$ & $\mathrm{NE}$ \\
\hline \multicolumn{5}{|l|}{ Pontederiaceae } \\
\hline Pontederia crassipes Mart. & $\sqrt{ }$ & & $\mathrm{H}$ & - \\
\hline \multicolumn{5}{|l|}{ Primulaceae } \\
\hline Ardisia complanata Wall. & & $\sqrt{ }$ & $\mathrm{S}$ & $\mathrm{NE}$ \\
\hline Embelia lampani Scheff. & & $\sqrt{ }$ & $\mathrm{C}$ & $\mathrm{NE}$ \\
\hline Embelia ribes Burm.f. & & $\sqrt{ }$ & $\mathrm{C}$ & $\mathrm{NE}$ \\
\hline Maesa sumatrana Scheff. & & $\sqrt{ }$ & $\mathrm{C}$ & $\mathrm{NE}$ \\
\hline \multicolumn{5}{|l|}{ Rhamnaceae } \\
\hline Ziziphus cf. elegans Wall. & & $\sqrt{ }$ & $\mathrm{C}$ & - \\
\hline \multicolumn{5}{|l|}{ Rhizophoraceae } \\
\hline Carallia brachiata (Lour.)Merr. & & $\sqrt{ }$ & $\mathrm{S}$ & $\mathrm{NE}$ \\
\hline \multicolumn{5}{|l|}{ Rubiaceae } \\
\hline Hynophytum formicarum Jack & $\sqrt{ }$ & & $\mathrm{H}$ & NE \\
\hline Ixora congesta Roxb. & & $\sqrt{ }$ & $\mathrm{S}$ & NE \\
\hline Ixora javanica (Blume) DC. & & $\sqrt{ }$ & $\mathrm{S}$ & $\mathrm{LC}$ \\
\hline Mitragyna speciosa Korth. & $\sqrt{ }$ & & $\mathrm{T}$ & $\mathrm{NE}$ \\
\hline Mussaenda frondosa $\mathrm{L}$. & & $\sqrt{ }$ & $\mathrm{S}$ & $\mathrm{NE}$ \\
\hline Nauclea officinalis (Pierre ex Pit.) Merr. \& Chun & $\sqrt{ }$ & & $\mathrm{T}$ & $\mathrm{NE}$ \\
\hline Neolamarckia cadamba (Roxb.) Bosser & & $\sqrt{ }$ & $\mathrm{T}$ & $\mathrm{NE}$ \\
\hline Porterandia anisophylla (Jack ex Roxb.) Ridl. & & $\sqrt{ }$ & $\mathrm{T}$ & $\mathrm{NE}$ \\
\hline Psychotria viridiflora Reinw. ex Blume & & $\sqrt{ }$ & $\mathrm{S}$ & $\mathrm{NE}$ \\
\hline Uncaria acida (W.Hunter) Roxb. & $\sqrt{ }$ & & $\mathrm{C}$ & NE \\
\hline Uncaria cordata (Lour.) Merr. & & $\sqrt{ }$ & $\mathrm{C}$ & $\mathrm{NE}$ \\
\hline \multicolumn{5}{|l|}{ Rutaceae } \\
\hline Luvunga sarmentosa (Blume) Kurz & & $\sqrt{ }$ & $\mathrm{C}$ & $\mathrm{NE}$ \\
\hline Melicope accedens (Blume) T.G.Hartley & & $\sqrt{ }$ & $\mathrm{T}$ & $\mathrm{NE}$ \\
\hline \multicolumn{5}{|l|}{ Selaginellaceae } \\
\hline Selaginella willdenowii (Desv.) Baker & & $\sqrt{ }$ & $\mathrm{H}$ & $\mathrm{NE}$ \\
\hline \multicolumn{5}{|l|}{ Simaroubaceae } \\
\hline Eurycoma longifolia Jack & & $\sqrt{ }$ & $\mathrm{S}$ & $\mathrm{NE}$ \\
\hline \multicolumn{5}{|l|}{ Smilacaceae } \\
\hline Smilax setosa Miq. & & $\sqrt{ }$ & $\mathrm{C}$ & $\mathrm{NE}$ \\
\hline \multicolumn{5}{|l|}{ Stemonuraceae } \\
\hline Cantleya corniculata (Becc.) R.A.Howard & & $\sqrt{ }$ & $\mathrm{T}$ & VU \\
\hline \multicolumn{5}{|l|}{ Theaceae } \\
\hline Eurya nitida Korth. & & $\sqrt{ }$ & $\mathrm{S}$ & NE \\
\hline Schima wallichii (DC.) Korth. & & $\sqrt{ }$ & $\mathrm{T}$ & LC \\
\hline \multicolumn{5}{|l|}{ Thelypteridaceae } \\
\hline Thelypteris decora (Domin.) C.F.Reed & $\sqrt{ }$ & & $\mathrm{H}$ & $\mathrm{NE}$ \\
\hline \multicolumn{5}{|l|}{$\begin{array}{l}\text { Thymelaeaceae } \\
\text { The }\end{array}$} \\
\hline Aquilaria hirta Ridl. & & $\sqrt{ }$ & $\mathrm{T}$ & VU \\
\hline \multicolumn{5}{|l|}{ Vitaceae } \\
\hline Ampelocissus thyrsiflora (Blume) Planch. & & $\sqrt{ }$ & $\mathrm{C}$ & $\mathrm{NE}$ \\
\hline Cayratia trifolia (L.) Mabb. \& J.Wen & $\sqrt{ }$ & & $\mathrm{C}$ & $\mathrm{NE}$ \\
\hline Leea indica (Burm.f.) Merr. & $\sqrt{ }$ & & $\mathrm{S}$ & $\mathrm{LC}$ \\
\hline Pterisanthes cissoides Blume & & $\sqrt{ }$ & $\mathrm{C}$ & $\mathrm{NE}$ \\
\hline Tetrastigma dichotomum (Miq.) Planch. & & $\sqrt{ }$ & $\mathrm{C}$ & $\mathrm{NE}$ \\
\hline \multicolumn{5}{|l|}{ Zingiberaceae } \\
\hline Globba aurantiaca Miq. & & $\sqrt{ }$ & $\mathrm{H}$ & NT \\
\hline
\end{tabular}

Note: MU: Muara Upu; SA: Simulak Anjing; LF: Life form; IUCN: Conservation status according to IUCN Red List where only native species are examined and any alien species are indicated with '-', species with uncertain identification (shown by cf.) also given as '-'; IDN: Protection by Indonesian Government No. 106/2018; C: H: herbs; S: shrub; SL: shrub-like; T: tree; TL: tree-like; NE: Not Evaluated; LC: Least Concern; NT: Near Threatened; VU: Vulnerable; EN: Endangered; CR: Critically Endangered

Dipterocarpus caudatus subsp. Penangianus in mainland Sumatra

Dipterocarpus caudatus contains two subspecies. The first is subsp. caudatus, a Philippines endemic, while the second is a more widespread subspecies, named subsp. penangianus. The recently collected specimens (specimens Mustaqim et al. 2309\&2315) from Simulak Anjing belonged to the subsp. penangianus, which differs from subsp. caudatus with $1.5-2.5 \mathrm{~cm}$ long petiole and applanate leaves (Figure 5J). Randi Agusti (2020 pers. comm.), stated that the subsp. penangianus has also been found in some areas of Sumatra, although, unfortunately, no formal publication has been made. This time, the sterile specimens collected from the Simulak Anjing have been identified as the subsp. penangianus, which is additional information for the geographical distribution of the species in mainland Sumatra. Before study, subsp. penangianus in Sumatra is formally known from two islands outside the mainland, i.e. Karimun and Mursala (Ashton 1978, 1982). Recent explorations showed that the subspecies still exist in Mursala Island, growing together with the recently rediscovered Dipterocarpus cinereus (Kusuma et al. 2013). Mursala is one of the closest islands to Simulak Anjing and, therefore, the knowledge of this species in Mursala- 
Simulak Anjing is now improved.

\section{Sumatra endemic plant species}

Three species in SAHF are endemic to Sumatra. The first one is Nepenthes sumatrana (Nepenthaceae) that has only been found in North to West Sumatra (Cheek and Jebb 2001). The species inhabits the lowland habitat up to 1500 $\mathrm{m}$ asl. (Cheek and Jebb 2001; Fadillah et al. 2014) and listed under IUCN (2020) as Critically Endangered species.
The second is shrubby Thottea tapanuliensis (Aristolochiaceae) (Figure 5K) which was also recently described in 2020. The species is endemic to Sumatra, and is only known from SAHF with the preliminary IUCN Red List conservation status is Critically Endangered (Mustaqim and Putra 2020). The third is Macrolenes tuberculata (Melastomataceae) (Figure 5L), a woody climber that has been described in 2019.

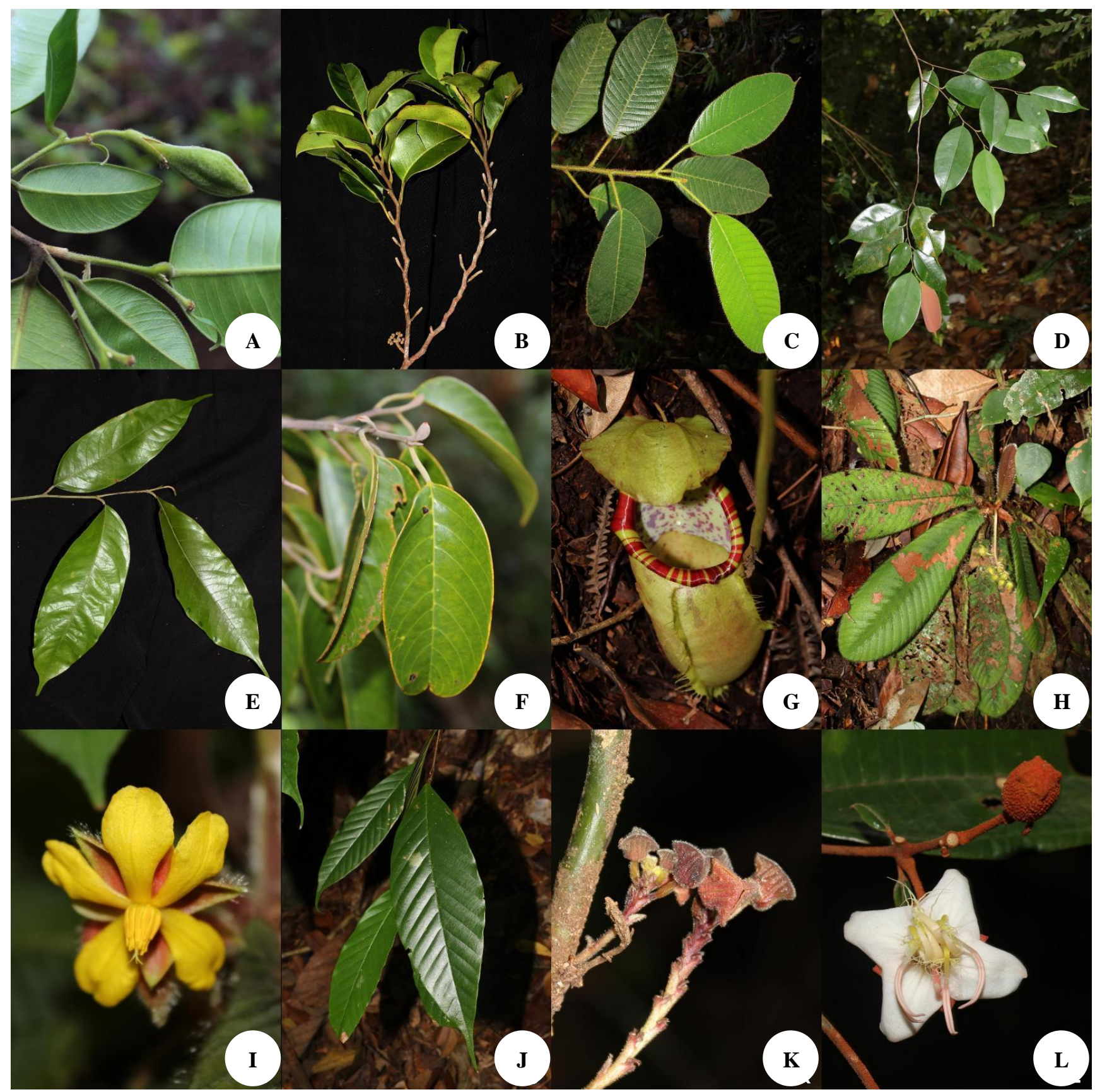

Figure 5. Selected plant species from Muara Upu Swamp Forest and Simulak Anjing Hill Forests, Batang Toru, North Sumatra, Indonesia: the Vulnerable species Aquilaria hirta (A), Cantleya corniculata (B), Dipterocarpus crinitus (C), and Dryobalanops sumatrensis (D); Endangered Vatica perakensis (E); Critically Endangered Shorea johorensis (F) and, also protected and endemic, Nepenthes sumatrana $(\mathrm{G})$; distribution confirmed in Sumatra for Acrotrema costatum (H-I); record for mainland Sumatra for Dipterocapus penangianus subsp. penangianus (J); as well as other two endemics Thottea tapanuliensis $(\mathrm{K})$ and Macrolenes tuberculata $(\mathrm{L})$ 
Table 2. Checklist of main NTFPs of plants according to PROSEA for the species in Muara Upu and Simulak Anjing, Batang Toru, North Sumatra, Indonesia

\begin{tabular}{lcl}
\hline Uses categories & $\begin{array}{c}\text { Number of } \\
\text { species }\end{array}$ & List of species \\
\hline Medicinal and poisonous & 63 & Acrostichum aureum, Acrotrema costatum, Alstonia spatulata, Anisophyllea disticha, \\
plants & Arcangelisia flava, Artocarpus elasticus, Asplenium nidus, Blechnum orientale, Breynia \\
& racemosa, Chromolaena odorata, Carallia brachiata, Cayratia trifolia, Cheilocostus \\
& speciosus, Cinnamomum iners, Cnestis palala, Colocasia esculenta, Commelina \\
& diffusa, Cyclea barbata, Cyperus iria, Derris trifoliata, Dicranopteris linearis, Dillenia \\
& indica, Dryobalanops sumatrensis, Elaeocarpus mastersii, Embelia ribes, \\
& Endospermum diadenum, Eurycoma longifolia, Ficus deltoidea, Ficus hispida, Ficus \\
& microcarpa, Ficus padana, Flagellaria indica, Gluta wallichii, Goniothalamus \\
& macrophyllus, Homalanthus populneus, Hydnophytum formicarum, Knema furfuracea, \\
& Leea indica, Lophopetalum javanicum, Macaranga bancana, Macaranga gigantea, \\
& Macaranga hypoleuca, Melastoma malabathricum, Mitragyna speciosa, Oncosperma \\
& horridum, Paraderris elliptica, Phanera semibifida, Pothos scandens, Psychotria \\
& viridiflora, Pterisanthes cissoides, Rhaphidophora korthalsii, Rhodamnia cinerea, \\
& Scaphium macropodum, Schima wallichii, Scleria sumatrensis, Spatholobus \\
& ferrugineus, Stenochlaena palustris, Tetracera indica, Trichosanthes costata, Uncaria \\
& acida, Urena lobata, and Vitex pinnata
\end{tabular}

Fiber plants

19

Vegetables

Edible fruits and nuts

Dye and tannin producing plants

Ornamental

Forages

Fern and fern allies

Auxiliary plants

Essential-oil plants

Plants producing exudates

Plants yielding non-seed carbohydrates

Rattans

Spices

Vegetable oils and fats
18 Acrostichum aureum, Artocarpus elasticus, Blechnum orientale, Commelina diffusa, Colocasia esculenta, Cheilocostus speciosus, Cayratia trifolia, Embelia ribes, Ficus fistulosa, Ficus grossularioides var. stenoloba, Leea indica, Melastoma malabathricum, Oncosperma tigillarium, Schismatoglottis calyptrata, Stenochlaena palustris, Trema cannabinum, Trichosanthes costata, and Uncaria acida

14 Artocarpus elasticus, Antidesma stipulare, Baccaurea parviflora, Castanopsis lucida, Dialium indum, Dryobalanops sumatrensis, Embelia ribes, Ficus hispida, Ficus padana, Leea indica, Mangifera griffithii, Pimelodendron griffithianum, Rhodamnia cinerea, and Trichosanthes costata

12 Arcangelisia flava, Castanopsis lucida, Dillenia indica, Durio griffithii, Dialium indum, Ficus padana, Macaranga gigantea, Melastoma malabathricum, Psychotria viridiflora, Rhodamnia cinerea, Syzygium zeylanicum, and Urena lobata

11 Acrostichum aureum, Asplenium nidus, Carallia brachiata, Dillenia indica, Ficus deltoidea, Ixora congesta, Melastoma malabathricum, Oncosperma horridum, Oncosperma tigillarium, Schismatoglottis calyptrata, and Stenochlaena palustris

7 Colocasia esculenta, Derris trifoliata, Ficus hispida, Ficus padana, Ottochloa nodosa,Schima wallichii, and Urochloa mutica

6 Acrostichum aureum Asplenium nidus, Blechnum orientale, Dicranopteris linearis, Drynaria quercifolia, Stenochlaena palustris, and Thelypteris decora

3 Adinandra dumosa Ficus hispida, and Schimawallichii

2 Cananga odorata and Dipterocarpus crinitus

2 Dyera costulata and Shorea acuminata

2 Colocasia esculenta and Cheilocostus speciosus

1 Calamus melanochaetes

1 Cinnamomum iners

$1 \quad$ Shorea leprosula 


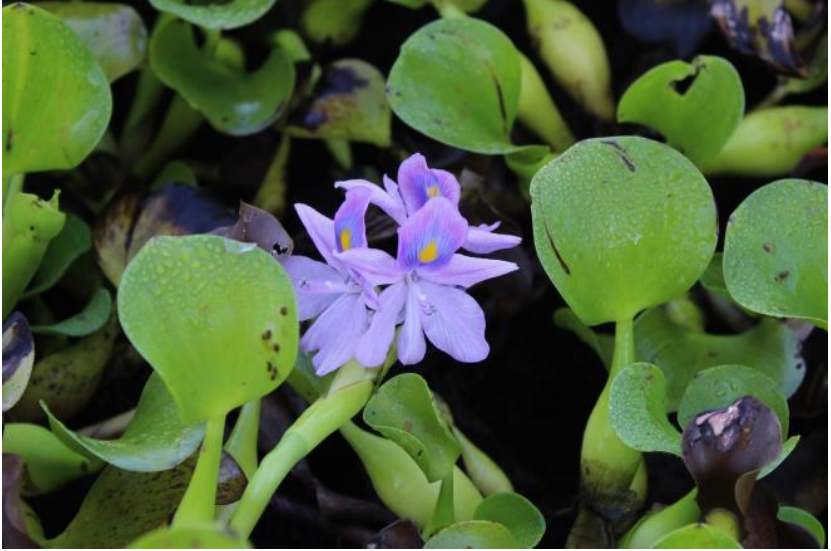

Figure 6. Flowering Pontederia crassipes in Muara Upu Swamp Forest, Batang Toru, North Sumatra

In Sumatra, the species is only known from Aceh and North Sumatra Provinces, with the previously southernmost record coming from Subulussalam, Aceh Province (Kartonagoro et al. 2019), positioned around 150 $\mathrm{km}$ north from SAHF. The presence of those three Sumatran endemic species increases the value of SAHF in the future conservation of lowland Sumatran rainforest diversity.

\section{Potential Non-Timber Forest Products (NTFPs) of the native species}

Many species in both areas are economically important, especially by the presence of many dipterocarp species which have valuable timber (Ashton 1982). However, this use should be avoided for SAHF or MUSF as they are quite small in area size. Therefore, numerous good alternatives can be acquired from the NTFPs such as medicine. Dryobalanops sumatrensis is also known as barus tree, stands among the most historically important species, and became an important commodity since the Dutch East Indie era. Besides the quality of the wood, the species was once the most important source of camphor which has excellent medicinal properties to cure many diseases like asthma, cough, stomach or liver pain, rheumatism, etc. (Lee et al. 1993). Besides that, Eurycoma longifolia or tongkat ali or known also as pasak bumi, is a very important medicinal plant, besides the long history of its value as a source of aphrodisiac. It has been proved that the chemical constituents of the species have excellent properties to improve human fertility (Mustaqim et al. 2020). These are two examples of the popular NTFPs yielding species. All species are checked using the PROSEA database (PROSEA 2020; see also Wulijarni-Soetjipto and del Rosario 2003) and listed according to the main uses. The results of the comparisons showed that 88 species have either single or multiple uses. Most of the plants have been used as medicine and source of poison with 63 species, followed by fiber with 19 species, vegetables with 18 species, and edible fruit or nuts with 14 species (Table 2).

\section{Alien plants}

There are 16 species of alien plants recorded in this study. Four of them belong to 100 of the most invasive species, i.e. Pontederia crassipes or water hyacinth (Figure 6), Clidemia hirta, or simply known as clidemia, Mimosa pigra or bashful plant, and Mikania micrantha or American rope (Lowe et al. 2000; GISD 2020). The presence of $P$. crassipes was recorded from MUSF, forming a dense floating population competing with the local species like Hanguana anthelminthica and Thelypteris decora. In the MUSF area, the potentially invasive climbing aroids Syngonium podophylllum has also been recorded. The species has been reported to grow as abundant naturalized species in Southeast Asia, i.e. Singapore (Chong et al. 2010), and Java (Mustaqim and Nisyawati 2016), while a new record of Melothria pendula (Cucurbitaceae) for Sumatra has also been published recently based on collections from MUSF (Mustaqim and Putra 2020b). Meanwhile, in the SAHF, a small population of M. pigra, one of the 100 most invasive species (Lowe et al. 2000), has been recorded in the margin of the remaining forest.

The presence of alien plant species in the peat swamps of North Sumatra's west coast has previously been documented. In the Tripa peat swamp forest, Arongan, Aceh Barat Regency, several species of alien plants were also recorded, including Lantana camara (Verbenaceae), alongside Piper aduncum (Piperaceae), Sphagneticola trilobata (Asteraceae), and several herbs or shrubs (Djufri and Samingan 2013). Lantana camara also belongs to the 100 most invasive species in the world (GISD 2020), and it has been found either from the disturbed or undisturbed peat swamp forests (Djufri and Samingan 2013). The results of this study confirmed that it is important to note the uniqueness of peat swamp forests (Giesen et al. 2018), alien species are still able to enter and establish themselves in peat swamp forests. The study observed that many alien species are limited to the disturbed areas of MUSF, except for the climbing $S$. podophyllum, a species native to tropical America (Croat 1981).

The presence of non-native alien species should be taken into consideration for all related stakeholders, where the eradication should be started as soon as possible. For the SAHF, the study showed a very low spread of nonnative species which supports the previous statement that alien species would not be able to enter more less disturbed vegetations (van Steenis 1972). However, a recent study found that this statement could not be applied to all species because many alien species could also become invasive in a shaded forest. Although this latter statement was based on the study in temperate forests, the possibility of the same situation could happen in a tropical rainforest, so that this should also be taken into consideration (Dyderski and Jagodziński 2018).

In conclusion, SAHF is a home for several endemic, poorly known, and/or threatened plant species, of which one of them just only been described recently. There are also two noteworthy records in Sumatra for Acrotrema costatum and Dipterocarpus penangianus subsp. penangianus. Compared to the published literature, many native plant species have valuable NTFPs that can be 
optimized for a sustainable conservation effort, with most species have been reported having medicinal values, sources of fiber, or even fruits. In the degraded peat swamp forest of Muara Upu, there are some non-native species that some of them have been reported as invasive elsewhere. This includes the notorious water hyacinth (Pontederia crassipes). Meanwhile, only a few non-native plants were recorded from Simulak Anjing Hill Forests. A proper eradication of the non-native plant species, especially ones that could become invasive, should be started as soon as possible to prevent further spread and minimize the negative impact on the native plants or other biotas.

\section{ACKNOWLEDGEMENTS}

This work was supported by Unilever and PTPN III via the Faculty of Agriculture, IPB University, Bogor, Indonesia c.q. Dr. Supijatno. The authors thanked all of Dr. Supijatno team members as well as staff from PTPN III Muara Upu, South Tapanuli, Indonesia for invaluable help during the fieldwork where the materials used in this paper have been collected. Also thanks to the Laboratory of Ecology and Plant Resources, Department of Biology, IPB University, for facilities during the preparation of the manuscript.

\section{REFERENCES}

Ashton PS. 1978. Flora Malesiana precursores: Dipterocarpaceae. Gard Bull Singap 31 (1): 5-48.

Ashton PS. 1982. Dipterocarpaceae. Fl Malesiana ser I. 9 (2): 237-552.

Cheek M, Jebb M. 2001. Nepenthaceae. Fl Malesiana ser I. 15: 1-157.

Chong KY, Ang PT, Tan HTW. Identity and spread of an exotic Syngonium species in Singapore. Nat Sing 3: 1-5.

Clarke C. 2001. Nepenthes of Sumatra and Peninsular Malaysia. Natural History Publications Borneo, Kota Kinabalu.

Clarke CM. 2014. Nepenthes sumatrana. The IUCN Red List of Threatened Species 2014: e.T39700A21845285. DOI: 10.2305/IUCN.UK.2014-1.RLTS.T39700A21845285.en.

Croat TB. 1981. A revision of Syngonium (Araceae). Ann Missouri Bot Gard 68 (4): 565-651. DOI: $10.2307 / 2398892$

Djufri, Samingan. 2013. Komposisi flora kawasan Rawa Tripa di Kabupaten Aceh Barat. J EduBio Trop 1 (1): 1-13. [Indonesian]

Dyderski MK, Jagodziński AM. 2018. Low impact of disturbance on ecological success of invasive tree and shrub species in temperate forests. Plant Ecol 219 (11): 1369-1380. DOI: 10.1007/s11258-0180885-4

Fadillah NN, Patana P, Yunasfi. 2014. Keanekaragaman Nepenthes pada kawasan kebun bonsai dan sekitarnya di Cagar Alam Dolok Sibual Buali, Sumatera Utara. Peronema For Sci J 3 (2): 1-8.

Giesen W, Wijedasa LS, Page SE. Unique Southeast Asian peat swamp forest habitats have relatively few distinctive plant species. Mires Peat 22: 1-13. DOI: 10.19189/MaP.2017.OMB.287.

GISD (Global Invasive Species Database). 2020. 100 of the World's Worst Invasive Alien http://www.iucngisd.org/gisd/100_worst.php.

Global Wetlands V3. 2020. https://www2.cifor.org/global-wetlands/

Grey-Wilson C. 1989. A revision of Sumatran Impatiens: Studies in Balsaminaceae: VIII. Kew Bull 44 (1): 67-106. DOI $10.2307 / 4114646$

Hoogland RD. 1951. Dilleniaceae. Fl Malesiana ser I. 4 (3): 141-174.
IUCN. 2012. IUCN Red List categories and criteria, version 3.1, $2^{\text {nd }}$ ed. IUCN, Gland/Cambridge.

IUCN. 2020. The IUCN Red List of Threatened Species. Version 2020-3. https://www.iucnredlist.org.

IUCNSPC (IUCN Standards and Petitions Committee). 2019. Guidelines for Using the IUCN Red List Categories and Criteria. Version 14. Prepared by the Standards and Petitions Committee. http://www.iucnredlist.org/documents/RedListGuidelines.pdf.

Kartonagoro A, Hovenkamp P, van Welzen PC. 2019. A taxonomic revision of Macrolenes (Melastomataceae). Gard Bull Singap 71 (1): 185-241. DOI: 10.26492/gbs71(1).2019-12

Kusuma YWC, Wihermanto, Risna RA, Ashton PS. 2013. Rediscovery of the supposedly extinct Dipterocarpus cinereus. Oryx 47 (3): 324-324. DOI: $10.1017 / \mathrm{S} 0030605313000756$

Laumonier Y, Uryu Y, Stüwe M, Budiman A, Setiabudi B, Hadian O. 2010. Eco-floristic sectors and deforestation threats in Sumatra: identifying new conservation area network priorities for ecosystembased land use planning. Biodivers Conserv 19 (4): 1153-1174. doi: 10.1007/s10531-010-9784-2.

Lee HS, Wong WC, Ilic J, Sosef MSM. 1993. Dryobalanops Gaertner f.. In: Soerianegara I, Lemmens RHMJ (eds.) Plant Resources of SouthEast Asia No 5 (1): Timber trees; Major commercial timbers. PROSEA Foundation, Bogor, Indonesia. Database record: prota4u.org/prosea.

Loucks C, Whitten T. 2020. Southeastern Asia: Island of Sumatra in Indonesia. https://www. worldwildlife.org/ecoregions/im0158. [24 December 2020].

Lowe S, Browne M, Boudjelas S, De Poorter M. 2000. 100 of the World's Worst Invasive Alien Species. A Selection from the Global Invasive Species Database. Invasive Species Specialist Group (ISSG), IUCN, Gland/Cambridge.

Middleton DJ, Armstrong K, Baba Y, Balslev H, Chayamarit K, Chung RCK, Conn BJ, Fernando ES, Fujikawa K, Kiew R, Luu HT, Mu Mu Aung, Newman MF, Tagane S, Tanaka N, Thomas DC, Tran TB, Utteridge TMA, van Welzen PC, Widyatmoko D, Yahara T, Wong KM. 2019. Progress on Southeast Asia's flora projects. Gard Bull Singap 71 (2): 267-319. DOI: 10.26492/gbs71(2).2019-02

Mustaqim WA, Nisyawati. 2016. Records of adventive Syngonium wendlandii (Araceae) from Universitas Indonesia, Depok, West Java. Aroideana 39 (3): 23-26.

Mustaqim WA, Putra HF. 2020a. Thottea tapanuliensis (Aristolochiaceae): A new species from Sumatra, Indonesia. Telopea 23: 163-168. DOI: $10.7751 /$ telopea14535

Mustaqim WA, Putra HF. 2020b. Melothria (Cucurbitaceae): A new genus record of naturalized cucumber in Sumatra. Floribunda 6 (5): 183-187. DOI: 10.32556/floribunda.v6i5.2020.318

Mustaqim WA, Raihandhany R, Tamam MB. 2020. Eurycoma longifolia Jack Simaroubaceae. In: Franco FM (ed) Ethnobotany of the Mountain Regions of Southeast Asia. Ethnobotany of Mountain Regions. Springer, Nederland. DOI: 10.1007/978-3-030-14116$5 \_162-1$

POWO. 2020. Plants of the world online. http://www.plantsoftheworldonline.org.

PROSEA. 2020. Plant Resources of Southeast Asia. http://www.prota4u.org/prosea/.

Rugayah, Retnowati A, Windradi FI, Hidayat A. 2004. Pengumpulan data taksonomi. In: Rugayah, Widjaja EA, Praptiwi (eds) Pedoman Pengumpulan Data Keanekaragaman Flora. Puslit Biologi LIPI, Bogor. [Indonesian]

Susatya A. 2011. Rafflesia pesona bunga terbesar di dunia. Direktorat Kawasan Konservasi dan Bina Hutan Lindung, Jakarta. [Indonesian]

Thiers B. 2020-continuously updated. Index Herbariorum: A global directory of public herbaria and associated staff. New York Botanical Garden's Virtual Herbarium. http://sweetgum.nybg.org/ih/

UNESCO. 2020. Tropical rainforest heritage of Sumatra. https://whc.unesco.org/en/list/1167/.

van Steenis CGGJ. 1972. The mountain flora of Java. EJ Brill, Leiden.

Wulijarni-Soetjipto N, del Rosario BP. 2003. PROSEA and Flora Malesiana. Telopea 10 (1): 11-27.

Yuzammi, Kurniawan A, Asih NPS, Erlinawati I, Hetterscheid W. 2017. The Amorphophallus of Indonesia. Center for Plant Conservation, Botanic Garden, Indonesia Institute of Science, Bogor. [Indonesian] 